\title{
A Real Test System For Power System Planning, Operation, and Reliability
}

\author{
Meisam Mahdavi ${ }^{1} \cdot$ Carlos Sabillón ${ }^{1} \cdot$ Majid Ajalli ${ }^{2} \cdot$ Hassan Monsef ${ }^{3} \cdot$ Ruben Romero $^{1}$
}

Received: 10 April 2017 / Revised: 17 November 2017 / Accepted: 27 December 2017 / Published online: 1 February 2018

(c) Brazilian Society for Automatics-SBA 2018

\begin{abstract}
Nowadays, several test systems available in the specialized literature are used to verify studies regarding power system planning or network reliability. However, there are no test systems currently available with enough information in order to endorse studies that simultaneously approach expansion planning, operation, and reliability issues. This paper introduces a real test system, including the load modeling, and generation and transmission systems. The main objective is to provide all the details and information required to evaluate methods and models developed for power system planning, operation, and reliability. The presented load modeling includes hourly, daily, weekly, monthly, and seasonal patterns. Furthermore, besides the substation data, reliability details, construction costs, and characteristics of right of ways (e.g., line length, impedance, and ratings) for the transmission system are exposed. The real transmission system presented contains 39 buses, 135 transformers, and 66 lines at two voltage levels: 230 and $400 \mathrm{kV}$. Finally, the generation system reliability data as well as operation and installation costs for each unit are also provided.
\end{abstract}

Keywords Network reliability $\cdot$ Power system operation $\cdot$ Power system planning $\cdot$ Real test system

\section{Introduction}

Interests in studying reliability and operation issues together with power system planning have increased due to the important role played by these areas in the generation and transmission capacity expansion. For reliability studies, test systems such as the RBTS (Billinton 1989), IEEE RTS (IEEE Reliability Test System 1979), and Korean southeast power system (Choi et al. 2006) have been introduced. On the other hand, Garver's network (Garver 1970), Brazilian 46, 78-, and 87-bus interconnections (Romero and Monticelli 1994; Romero et al. 2002), Colombian network (Escobar 2002; Escobar et al. 2004), IEEE 24-, 25-, and 30-bus systems (Ekwue and Cory 1984; Fang and Hill 2003; Tor et al.

Ruben Romero

ruben@dee.feis.unesp.br

1 Department of Electrical Engineering, Faculty of Engineering on Ilha Solteira Campus, São Paulo State University, Ilha Solteira, SP, Brazil

2 Department of Electrical Engineering, Shahid Beheshti University, Tehran, Iran

3 School of Electrical and Computer Engineering, College of Engineering, University of Tehran, Tehran, Iran
2010), Portuguese generation/transmission network (Braga and Saraiva 2005), and Iranian 18-bus regional and $400 \mathrm{kV}$ national grids (Shayeghi and Mahdavi 2009; Maghouli et al. 2011) have been used for planning case studies. However, none of these case studies provides a simultaneous comparison of the results obtained using different methods of planning, operation, and reliability. Thus, it is desirable to have a reference test system that incorporates the real basic data needed in power system planning, operation, and reliability evaluation.

In existing test systems, the whole set of parameters needed for both planning and reliability applications is not provided. For example, Garver's network and other case studies about power system planning (Garver 1970; Romero and Monticelli 1994; Romero et al. 2002; Escobar 2002; Escobar et al. 2004; Ekwue and Cory 1984; Fang and Hill 2003; Tor et al. 2010; Braga and Saraiva 2005; Shayeghi and Mahdavi 2009; Maghouli et al. 2011) do not contain reliability and operation details. Moreover, reliability test systems as reported in (Billinton 1989; IEEE Reliability Test System 1979; Choi et al. 2006) do not include configuration and complete reliability information about substations [e.g., number, age and capacity of transformers at each bus, as well as their forced outage rate (FOR), forced outage duration (FOD), 
Table 1 Seasonal peak load as percentages of annual peak

\begin{tabular}{lllll}
\hline Season $(\%)$ & Winter $(\%)$ & Spring $(\%)$ & Summer $(\%)$ & Autumn $(\%)$ \\
\hline Peak load & 91.3 & 90.6 & 100 & 90.2 \\
\hline
\end{tabular}

Table 2 Monthly peak load as percentages of seasonal peak

\begin{tabular}{llllll}
\hline Season & Month & Peak load $(\%)$ & Season & Month & Peak load $(\%)$ \\
\hline \multirow{2}{*}{ Winter } & Jan. & 99.3 & Summer & Jul. & 98.2 \\
& Feb. & 97.8 & & Aug. & Sep. \\
\multirow{3}{*}{ Spring } & Mar. & 100 & Autumn & Oct. & 94.1 \\
& Apr. & 85.9 & & Nov. & 89.6 \\
& May & 90.4 & & Dec. & 88.5 \\
\hline
\end{tabular}

Table 3 Weekly peak load as percentages of annual peak (\%)

\begin{tabular}{|c|c|c|c|c|c|c|c|c|c|c|c|c|c|c|c|}
\hline W. & Peak load & W. & Peak load & W. & Peak load & W. & Peak load & W. & Peak load & W. & Peak load & W. & Peak load & W. & Peak load \\
\hline 1 & 81.5 & 8 & 82.2 & 15 & 75.9 & 22 & 85 & 29 & 99.2 & 36 & 94 & 43 & 77.6 & 50 & 81.65 \\
\hline 2 & 81.8 & 9 & 81.7 & 16 & 77.2 & 23 & 88.6 & 30 & 98.4 & 37 & 92.6 & 44 & 80 & 51 & 81.5 \\
\hline 3 & 80 & 10 & 81.35 & 17 & 79 & 24 & 90.7 & 31 & 99 & 38 & 91 & 45 & 80.5 & 52 & 81.2 \\
\hline 4 & 81.2 & 11 & 81.95 & 18 & 80 & 25 & 93.8 & 32 & 100 & 39 & 90.2 & 46 & 80 & - & - \\
\hline 5 & 81.5 & 12 & 75.6 & 19 & 82 & 26 & 93.2 & 33 & 98.95 & 40 & 85.7 & 47 & 80.4 & - & - \\
\hline 6 & 79.4 & 13 & 67.85 & 20 & 83.2 & 27 & 96.1 & 34 & 97.9 & 41 & 84.6 & 48 & 79.9 & - & - \\
\hline 7 & 81.4 & 14 & 74.4 & 21 & 85.8 & 28 & 97.3 & 35 & 96 & 42 & 81.8 & 49 & 80.6 & - & - \\
\hline
\end{tabular}

and scheduled outage duration (SOD)]. Furthermore, data on future expansion (e.g., load growth and lengths of new right of ways considering geographical limitations), actual location of buses, line characteristics (e.g., bundled conductors and line types), reliability and construction costs, daily peak loads, load diversity between buses, and scheduled outages of transmission lines such as repair rate and mean time to repair (MTTR) were also ignored. Besides, data regarding equipment age and substations configurations, which have important effects on planning decisions (Mahdavi et al. 2016), have not been considered by proposed test systems such as RTS. Reliability parameters such as generators forced and scheduled outage duration, lines MTTR and repair rate, and transformers forced and scheduled outages could efficiently affect proposed expansion plans. However, these essential data have not provided by RTS and other reliability test systems.

The main contribution of this work is to provide all the details and information required to evaluate methods and models developed for power system planning, operation, and reliability; i.e., gathering the different data required for studying an experimental power system from various aspects. It describes the reliability data, generation system characteris- tics, and transmission network details. This paper provides essential data on the expansion, operation, and reliability of an actual power system, which is part of the Iranian north interconnected network and well-known as the regional electric company of Tehran (RECT). All expansion, operation, and reliability data, except for lost load and energy costs, are representative of the experiences of the RECT. The values for lost loads and costs of energy not supplied are based on reliability data from Canada, because such expenses have not yet been calculated in Iran. This case study does not include distribution system configuration or protective relays data, because the aim is to define a system that is broad enough to provide a basis for reporting on analysis methods for combined generation/transmission expansion planning with composite power system reliability.

\section{Description of Test System}

\subsection{Load Characteristics}

The annual peak load for the test system is 10729 MW. Table 1 describes the peak loads as a percentage of the annual 
Table 4 Daily peak load as percentages of weekly peak (\%)

\begin{tabular}{|c|c|c|c|c|c|c|c|c|c|c|c|c|c|}
\hline \multirow[t]{2}{*}{ Days } & \multicolumn{13}{|c|}{ Week number } \\
\hline & 1 & 2 & 3 & 4 & 5 & 6 & 7 & 8 & 9 & 10 & 11 & 12 & 13 \\
\hline Mon & 98.6 & 100 & 99.95 & 99.4 & 100 & 100 & 98.2 & 100 & 98.3 & 99.5 & 100 & 100 & 100 \\
\hline Tue & 98.3 & 97.4 & 99.6 & 99.8 & 99 & 94.95 & 98.9 & 98.6 & 99.1 & 98.5 & 98.2 & 80.4 & 99.795 \\
\hline Wed & 99.3 & 89.4 & 98.7 & 99.8 & 99.4 & 82.1 & 98.6 & 99.5 & 99.3 & 99.4 & 97.6 & 83.6 & 99.4 \\
\hline Thu & 95 & 92.6 & 91.2 & 94.7 & 95.3 & 80.3 & 95.2 & 94.4 & 95.5 & 96.6 & 95.3 & 83.9 & 99.2 \\
\hline Fri & 90.3 & 87.8 & 89.7 & 89.5 & 90 & 88.6 & 90.5 & 89.7 & 92.8 & 92.35 & 91 & 83.8 & 96 \\
\hline Sat & 99.1 & 96.2 & 99.9 & 98.8 & 97.8 & 92.6 & 99.5 & 97.75 & 100 & 100 & 94.7 & 85.1 & 95.4 \\
\hline Sun & 100 & 96.6 & 100 & 100 & 96.6 & 99.3 & 100 & 97.9 & 98.5 & 99.4 & 93.2 & 89.6 & 93.5 \\
\hline Days & 14 & 15 & 16 & 17 & 18 & 19 & 20 & 21 & 22 & 23 & 24 & 25 & 26 \\
\hline Mon & 98 & 99.5 & 99.1 & 98.5 & 98.3 & 96.75 & 97.8 & 95.5 & 99.6 & 93.97 & 96 & 98.3 & 99.8 \\
\hline Tue & 98 & 98 & 100 & 100 & 100 & 97.65 & 97.5 & 96.6 & 97.4 & 100 & 98.6 & 99.5 & 96.6 \\
\hline Wed & 98.8 & 99.9 & 98.6 & 95.3 & 98 & 98.5 & 100 & 96.45 & 99.7 & 98 & 98.2 & 99.2 & 97.5 \\
\hline Thu & 100 & 96.7 & 99.6 & 98 & 98 & 96.5 & 99.7 & 97.1 & 100 & 95.9 & 89.1 & 99.5 & 98.8 \\
\hline Fri & 96.45 & 97.5 & 98.6 & 97.6 & 95.4 & 98.4 & 97 & 93.9 & 98.95 & 94.6 & 98.2 & 94.2 & 95.2 \\
\hline Sat & 90.6 & 95 & 95.3 & 91.5 & 89.4 & 93 & 92 & 91.8 & 93.8 & 91.9 & 95.2 & 92.6 & 92.9 \\
\hline Sun & 95.7 & 100 & 99.9 & 98.7 & 96.4 & 100 & 98.65 & 100 & 95.3 & 98.7 & 100 & 100 & 100 \\
\hline Days & 27 & 28 & 29 & 30 & 31 & 32 & 33 & 34 & 35 & 36 & 37 & 38 & 39 \\
\hline Mon & 98.6 & 95.1 & 100 & 97.9 & 100 & 100 & 98.65 & 98.8 & 100 & 99.45 & 100 & 100 & 99 \\
\hline Tue & 98.1 & 99.6 & 99.7 & 98.95 & 99.7 & 99.2 & 99.65 & 98.5 & 99.5 & 100 & 99.8 & 98.7 & 100 \\
\hline Wed & 100 & 94.8 & 98.9 & 98.5 & 98.35 & 98.3 & 100 & 98.7 & 96 & 97.5 & 98.9 & 93.345 & 98.2 \\
\hline Thu & 99.9 & 98.4 & 97.6 & 98.6 & 98.9 & 97 & 97.7 & 96 & 95.7 & 97.3 & 98.4 & 99.1 & 96 \\
\hline Fri & 96.6 & 97.3 & 95.75 & 96.5 & 95 & 96 & 95.65 & 90.9 & 93.4 & 93.5 & 94.3 & 96.945 & 92.7 \\
\hline Sat & 92.9 & 93.8 & 91.1 & 92.4 & 93.65 & 89.9 & 95.8 & 90.9 & 90.7 & 89.8 & 89.7 & 91.9 & 88.1 \\
\hline Sun & 97.5 & 100 & 97.7 & 100 & 98.7 & 97.1 & 99.8 & 98 & 98.8 & 98.6 & 97.7 & 99.9 & 95.3 \\
\hline Days & 40 & 41 & 42 & 43 & 44 & 45 & 46 & 47 & 48 & 49 & 50 & 51 & 52 \\
\hline Mon & 100 & 98.9 & 99.6 & 99.1 & 97.5 & 99.8 & 98.35 & 99 & 96.9 & 99 & 99.1 & 99.2 & 98.3 \\
\hline Tue & 100 & 98.5 & 98.9 & 94.2 & 96.8 & 100 & 98.6 & 97.7 & 91.6 & 100 & 100 & 100 & 98 \\
\hline Wed & 99.3 & 100 & 99 & 89 & 93.8 & 97.3 & 99.1 & 97.8 & 98 & 99.8 & 99.4 & 99.7 & 98.9 \\
\hline Thu & 97.8 & 99.4 & 100 & 98.4 & 96.3 & 98 & 98.4 & 99.7 & 99.25 & 99.8 & 99 & 96.85 & 100 \\
\hline Fri & 95.8 & 96 & 95.5 & 95.25 & 94.2 & 94.6 & 95.75 & 96.4 & 95.9 & 96.2 & 96.4 & 88.9 & 95.84 \\
\hline Sat & 91.5 & 91 & 89.7 & 92.95 & 87.4 & 89.25 & 89.9 & 88.3 & 90.3 & 88.5 & 88.9 & 90 & 89.1 \\
\hline Sun & 99.2 & 96.4 & 95.7 & 100 & 100 & 98.6 & 100 & 100 & 100 & 99.8 & 99.9 & 98.1 & 99.45 \\
\hline
\end{tabular}

peak load for each season. This table is helpful for multistage power system expansion planning when the planners consider a multi-level load for the electrical power demand. Table 2 shows monthly peak loads as a percentage of the seasonal peak loads for each month.

Three months in every season have been normalized with respect to the peak load of the corresponding season. For example, the peak load of January is stated as a percentage of winter peak load, and July is represented as a percentage of summer peak. Table 3 gives data on weekly peak loads as percentages of the annual peak load. Week 1 is taken the first week in January. The annual peak occurs at week 32.
Table 4 lists daily peak loads as percentages of the weekly peak. The data in Table 4 define a daily peak load model of $52 \times 7=364$ days, with Monday as the first day of the year.

Table 5 gives hourly load models for each of the four seasons. The first column reflects winter, while the second, third, and fourth columns indicate spring, summer, and autumn, respectively. Combining Tables 3, 4, and 5 with the annual peak load generates an hourly load model of $364 \times 24=8736$ h. In simple terms, the annual load curve for 8736 hours is available and the planners can determine minimum and maximum load level in every day, week, month, season or year. 
Table 5 Hourly load as percentages of daily peak (\%)

\begin{tabular}{|c|c|c|c|c|}
\hline Hour & Winter & Spring & Summer & Autumn \\
\hline 1 & 73 & 81 & 83.7 & 75 \\
\hline 2 & 70 & 78.7 & 80.3 & 73.5 \\
\hline 3 & 68 & 74 & 77.9 & 71 \\
\hline 4 & 69 & 70 & 75.6 & 69 \\
\hline 5 & 70 & 69 & 75 & 70 \\
\hline 6 & 74 & 70.5 & 74.4 & 71.5 \\
\hline 7 & 78 & 70 & 70.9 & 72 \\
\hline 8 & 72 & 75 & 70.3 & 79 \\
\hline 9 & 78 & 81 & 73.8 & 84 \\
\hline 10 & 79 & 85 & 77.9 & 89 \\
\hline 11 & 80 & 88 & 81.4 & 90 \\
\hline 12 & 85 & 90 & 85.5 & 91.5 \\
\hline 13 & 85 & 89 & 88.3 & 91 \\
\hline 14 & 80 & 86 & 89.5 & 88 \\
\hline 15 & 81 & 84 & 90.7 & 81.5 \\
\hline 16 & 87 & 87 & 91.3 & 83 \\
\hline 17 & 98 & 92 & 90.7 & 93 \\
\hline 18 & 100 & 96 & 87.2 & 96 \\
\hline 19 & 99 & 98 & 82.5 & 100 \\
\hline 20 & 98 & 100 & 83 & 98 \\
\hline 21 & 98.5 & 98.5 & 96 & 97 \\
\hline 22 & 94 & 97 & 100 & 96 \\
\hline 23 & 89 & 96 & 97 & 85 \\
\hline 24 & 80 & 87 & 92.5 & 81 \\
\hline
\end{tabular}

Minimum load would be useful for demand response and peak load shifting studies. The annual load factor for this model is 65 . The annual load factor is equal to the annual average demand divided by the annual peak load.

\subsection{Generation System}

Table 6 lists the generating unit ratings, the reduced-capacity duration (RCD), and reliability data (such as FOR, mean time to failure (MTTF), MTTR, FOD, and SOD), where RCD is the time that a generating unit is operated in derated state and can deliver partial output. FOD is the average time taken to repair the failed unit, i.e., FOD refers to the time necessary to execute corrective repairs, due to unexpected failures in a generating unit. On the other hand, SOD is the average duration of the time necessary to execute preventive repairs (maintenance), i.e., the generating unit was still working but a scheduled withdraw is done to correct specific defects in order to avoid forced outages (Mahdavi et al. 2017).

Table 7 gives the operating data for the generating units, while the unit size and operating output of the generation mix are shown in Table 8. Table 9 gives the ages and the installation costs of the generating units for maintenance and planning applications. Moreover, fuel costs are suggested in Tables 10 and 11. These costs are subject to variation due to geographical location and other factors. Thus, in Table 10 , nodal fuel transportation costs for power production are given.

Finally, the generating unit operating costs (OCs), commonly used in economic dispatch studies, can be calculated using the information presented in Tables 7, 10, 11, and 12 . The calculation of the OCs is shown in (1), in terms of the fuel rate (FR; fourth column of Table 7), the heat rate (HR; sixth column of Table 7), the Transportation Cost (TC; third column of Table 10), the Toll (fourth column of Table 10), and the Price (second column of Table 11). Further information regarding capacity outage for the generating units is presented as an "Appendix".

$\mathrm{OC}=\mathrm{FR} \times \mathrm{HR} \times(\mathrm{TC}+$ Toll + Price $)$

\subsection{Transmission Network}

The transmission network consists of 39 bus locations connected by 66 lines as shown in Fig. 1. The transmission lines are at two voltage levels: 230 and $400 \mathrm{kV}$. The locations of the generating units are shown in Table 12. Moreover, Table 12 shows the number of existing units in each generation bus and the maximum number of units (i.e., the sum of existing units and new units that can be installed in each generation bus).

It can be seen that 13 out of the 39 buses are generating stations. Moreover, buses 7 and 32 are connected to the Iranian Interconnected Network (which is a large transmission system with more than 200 buses); hence, they are considered slack buses. Table 13 provides data on generating unit reactive power capability for use in AC load flow calculations. Table 14 gives the reactive capability of voltage corrective devices. These devices help the system to maintain its rated voltage under contingency conditions. In addition, the annual peak load of the system is shown in Table 15.

In Table 15, the load diversity between buses is provided by load type. Load types 1, 2, 3, 4, and 5 indicate domestic, public, agricultural, industrial, and commercial demands, respectively. Moreover, substation characteristics, such as the number and capacity of each transformer, and their reliability data are given in Table 16. It is important to remark that the reliability information presented in Table 16 includes all the devices within the substation (e.g., transformers, capacitors, and reactors). In buses with the two transmission voltage levels $(5,8,12,13,19,25$, and 27), loads are connected to the $63 \mathrm{kV}$ side. The value of lost load (VOLL) and cost of energy not supplied (cost of ENS) for each load type are given in Table 17. The VOLL of each bus was obtained by combining the data in Table 15 with Table 17. For example, the VOLL of bus 1 is equal to $0.5 \times 150+0.28 \times 500+0.02 \times 3500+$ 
Table 6 Generating unit reliability data

\begin{tabular}{|c|c|c|c|c|c|c|c|}
\hline Unit size (MW) & No. of units & FOR & MTTF (h) & MTTR (h) & FOD (h) & SOD (h) & $\mathrm{RCD}(\mathrm{h})$ \\
\hline 12.5 & 4 & 0.036 & 1607 & 60 & 6 & 14 & 2553 \\
\hline 23.7 & 16 & 0.17 & 244 & 50 & 4.5 & 16 & 2941 \\
\hline 24 & 4 & 0.073 & 1016 & 80 & 13 & 20 & 6031 \\
\hline 25 & 5 & 0.17 & 195 & 40 & 6 & 9.5 & 2941 \\
\hline 32 & 13 & 0.17 & 146.5 & 30 & 7 & 8.5 & 2941 \\
\hline 38.5 & 3 & 0.072 & 902 & 70 & 10 & 22 & 6031 \\
\hline 82.5 & 3 & 0.08 & 460 & 40 & 6.5 & 8.5 & 1920 \\
\hline 85 & 3 & 0.017 & 2024 & 35 & 4 & 11 & 2941 \\
\hline 100 & 2 & 0.06 & 1097 & 70 & 3 & 4 & 1004 \\
\hline 102 & 3 & 0.019 & 2840 & 55 & 14.5 & 30 & 1655 \\
\hline 105 & 3 & 0.017 & 3469 & 60 & 6 & 13 & 2070 \\
\hline 116.2 & 6 & 0.019 & 1549 & 30 & 5 & 17 & 1655 \\
\hline 123.8 & 6 & 0.17 & 220 & 45 & 7 & 9 & 2070 \\
\hline 128.5 & 4 & 0.06 & 940 & 60 & 3 & 3 & 1004 \\
\hline 156.5 & 4 & 0.09 & 607 & 60 & 4 & 4 & 1090 \\
\hline 159 & 12 & 0.022 & 1778 & 40 & 8 & 22 & 2930 \\
\hline 161 & 4 & 0.15 & 340 & 60 & 8 & 15 & 5324 \\
\hline 166 & 5 & 0.04 & 960 & 40 & 8 & 10 & 980 \\
\hline 250 & 4 & 00.005 & 7960 & 40 & 11 & 3.8 & 2070 \\
\hline 263 & 3 & 0.09 & 708 & 70 & 3 & 7 & 1050 \\
\hline 318 & 5 & 0.035 & 2206 & 80 & 10 & 18 & 3240 \\
\hline 322 & 1 & 0.15 & 567 & 100 & 10 & 20 & 980 \\
\hline 332 & 1 & 0.04 & 2400 & 100 & 10 & 20 & 5324 \\
\hline 526 & 3 & 0.095 & 1429 & 150 & 10 & 30 & 1050 \\
\hline
\end{tabular}

$0.09 \times 1500+0.11 \times 4500=915 \$ / M W$. The cost of ENS for bus 39 is $4.17 \$ / \mathrm{kWh}(0.455 \times 0.56+0.15 \times 1.45+$ $0.05 \times 14+0.245 \times 5.5+0.1 \times 16.5)$. Furthermore, Table 18 illustrates the average annual load growth of each bus.

Figure 1 defines the actual geographical connections for the transmission network. The line lengths, which are shown in Tables 19 and 20, determine the physical bus locations in Fig. 1. Table 19 includes the number of circuits and bundled conductors, line voltage levels and types, transmission reliability data, and line ages for an actual network.

Line ages show when a transmission line was constructed in the network. Types 1, 2, and 3 indicate Canary, Cardinal, and Curlew conductors, while type 4 explains that the connection between the two buses is provided by cables. The length of each corridor is not a direct route between two buses. All distances have been calculated considering geographical limitations such as hills, forests, parks, roads, highways, farms, and other barriers. Table 20 gives the practical lengths of all candidate corridors for transmission network expansion, considering geographical restrictions. Impedance, rating data and construction cost for the lines are listed in Table 21.
The RECT system represents the 23 and $20 \%$ of the Iranian interconnected network total generation and consumption, respectively. Besides, its transmission and distribution power losses are 3.5 and $13.5 \%$ of the interconnected network power losses, respectively. The RECT system is the first ranked network in power generation and demand among all regional electric companies in Iran. In addition, each year the RECT system sales $5966 \mathrm{MWh}$ and buys $3777 \mathrm{MWh}$ to/from the interconnected network. Furthermore, annual average outage durations of 400 and $230 \mathrm{kV}$ lines in RECT system are 7.2 and 7.7 h, respectively. Finally, average duration of each outage for 400 and $230 \mathrm{kV}$ substations in RECT system is 5.9 and $30.5 \mathrm{~h}$, respectively.

\section{Conclusion}

A real test system known as regional electric company of Tehran (RECT) system, which is part of the Iranian north interconnected network, has been presented in this work, including the reliability data, generation system characteristics, and transmission network details. The presented data allow to incorporate various real parameters into experimental integrated models, contributing to the research on power 
Table 7 Generating unit operating data

\begin{tabular}{|c|c|c|c|c|c|}
\hline$\underline{\text { Size MW }}$ & Type & Fuel type & Fuel rate lit/kcal & Max. output MW & Heat rate $\mathrm{kcal} / \mathrm{kW}$ \\
\hline \multirow[t]{2}{*}{12.5} & Fossil & Oil & 1.0114 & 10 & 3958 \\
\hline & Steam & & & & \\
\hline \multirow[t]{2}{*}{23.7} & Comb. & Oil & 0.8566 & 20 & 3702 \\
\hline & Turbine & Gasoil & 0.1463 & & \\
\hline 24 & Hydro & - & - & 24 & - \\
\hline \multirow[t]{2}{*}{25} & Comb. & Oil & 0.8566 & 20 & 3702 \\
\hline & Turbine & Gasoil & 0.1463 & & \\
\hline \multirow[t]{2}{*}{32} & Comb. & Oil & 0.8566 & 26 & 3702 \\
\hline & Turbine & Gasoil & 0.1463 & & \\
\hline 38.5 & Hydro & - & - & 38.5 & - \\
\hline \multirow[t]{3}{*}{82.5} & Fossil & Gasoil & 0.0073 & 75 & 2960 \\
\hline & Steam & Gas & $0.7356^{*}$ & & \\
\hline & & Oil & 0.237 & & \\
\hline \multirow[t]{2}{*}{85} & Comb. & Oil & 0.8566 & 70 & 3702 \\
\hline & Turbine & Gasoil & 0.1463 & & \\
\hline \multirow[t]{2}{*}{100} & Combined & Gasoil & 0.093 & 100 & 1874 \\
\hline & Cycle & Gas & $0.9123^{*}$ & & \\
\hline \multirow[t]{2}{*}{102} & Combined & Gasoil & 1.481 & 100 & 1875 \\
\hline & Cycle & Gas & $0.853^{*}$ & & \\
\hline \multirow[t]{2}{*}{105} & Combined & Gasoil & 0.0763 & 100 & 1987 \\
\hline & Cycle & Gas & $0.93 *$ & & \\
\hline \multirow[t]{2}{*}{116.2} & Combined & Gasoil & 1.481 & 96.5 & 1875 \\
\hline & Cycle & Gas & $0.853^{*}$ & & \\
\hline \multirow[t]{2}{*}{123.8} & Combined & Gasoil & 0.0763 & 100 & 1987 \\
\hline & Cycle & Gas & $0.93 *$ & & \\
\hline \multirow[t]{2}{*}{128.5} & Combined & Gasoil & 0.093 & 106 & 1874 \\
\hline & Cycle & Gas & $0.9123^{*}$ & & \\
\hline \multirow[t]{3}{*}{156.5} & Fossil & Gasoil & 0.0075 & 150 & 2502 \\
\hline & Steam & Gas & $0.642^{*}$ & & \\
\hline & & Oil & 0.319 & & \\
\hline \multirow[t]{2}{*}{159} & Comb. & Oil & 0.77 & 135 & 3063 \\
\hline & Turbine & Gasoil & 0.231 & & \\
\hline \multirow[t]{2}{*}{161} & Combined & Gasoil & 0.0898 & 160 & 1850 \\
\hline & Cycle & Gas & $0.662 *$ & & \\
\hline \multirow[t]{2}{*}{166} & Comb. & Oil & 0.395 & 160 & 3500 \\
\hline & Turbine & Gasoil & 0.0576 & & \\
\hline \multirow[t]{3}{*}{250} & Fossil & Gasoil & 0.00007 & 250 & 2124 \\
\hline & Steam & Gas & $0.661^{*}$ & & \\
\hline & & Oil & 0.31 & & \\
\hline \multirow[t]{2}{*}{263} & Comb. & Oil & 0.149 & 250 & 3700 \\
\hline & Turbine & & & & \\
\hline \multirow[t]{2}{*}{318} & Comb. & Oil & 0.631 & 300 & 4000 \\
\hline & Turbine & & & & \\
\hline \multirow[t]{2}{*}{322} & Combined & Gasoil & 0.0898 & 320 & 1850 \\
\hline & Cycle & Gas & $0.662 *$ & & \\
\hline \multirow[t]{2}{*}{332} & Comb. & Oil & 0.395 & 320 & 3500 \\
\hline & Turbine & Gasoil & 0.0576 & & \\
\hline 526 & Comb. & Oil & 0.149 & 500 & 3700 \\
\hline & Turbine & & & & \\
\hline
\end{tabular}

$* \mathrm{~m}_{3} / \mathrm{kcal}$ 
Table 8 Generation mix

\begin{tabular}{lll}
\hline Type & Unit size (MW) & Operating output (MW) \\
\hline Fossil steam & 1923.5 & 1865 \\
Combustion turbine & 8202.2 & 7458 \\
Combined cycle & 3741 & 3363 \\
Hydro & 211.5 & 211.5 \\
Total & $14,078.2$ & 12897.5 \\
\hline
\end{tabular}

Table 9 Unit ages (year) and installation costs (Million\$)

\begin{tabular}{lrrlrl}
\hline Size MW & Age & Cost & Size MW & Age & Cost \\
\hline 12.5 & 56 & 10 & 123.8 & 21 & 111 \\
23.7 & 37 & 20 & 128.5 & 22 & 116 \\
24 & 9 & 14 & 156.5 & 42 & 125 \\
25 & 37 & 21 & 159 & 10 & 135 \\
32 & 37 & 27 & 161 & 6 & 145 \\
38.5 & 27 & 23 & 166 & 6 & 141 \\
82.5 & 47 & 66 & 250 & 23 & 200 \\
85 & 37 & 72 & 263 & 8 & 224 \\
100 & 17 & 90 & 318 & 9 & 270 \\
102 & 15 & 92 & 322 & 6 & 290 \\
105 & 14 & 95 & 332 & 5 & 282 \\
116.2 & 23 & 105 & 526 & 8 & 447 \\
\hline
\end{tabular}

Table 10 Fuel expenses

\begin{tabular}{|c|c|c|c|}
\hline Bus & Fuel & Transportation cost & Toll \\
\hline \multirow[t]{2}{*}{4} & Gas & $0.000004\left(\$ / \mathrm{m}^{3}\right)$ & $0.000058\left(\$ / \mathrm{m}^{3}\right)$ \\
\hline & Gasoil & 0.0000078 (\$/lit) & 0.00011 (\$/lit) \\
\hline \multirow[t]{3}{*}{7} & Gas & $0.0000052\left(\$ / \mathrm{m}^{3}\right)$ & $0.000058\left(\$ / \mathrm{m}^{3}\right)$ \\
\hline & Oil & 0.0000049 (\$/lit) & 0.000055 (\$/lit) \\
\hline & Gasoil & 0.0000097 (\$/lit) & 0.00011 (\$/lit) \\
\hline \multirow[t]{3}{*}{10} & Gas & $0.0000035\left(\$ / \mathrm{m}^{3}\right)$ & $0.000093\left(\$ / \mathrm{m}^{3}\right)$ \\
\hline & Oil & 0.0000033 (\$/lit) & 0.000089 (\$/lit) \\
\hline & Gasoil & 0.0000066 (\$/lit) & 0.00018 (\$/lit) \\
\hline \multirow[t]{2}{*}{11} & Gas & $0.0000035\left(\$ / \mathrm{m}^{3}\right)$ & $0.000093\left(\$ / \mathrm{m}^{3}\right)$ \\
\hline & Gasoil & 0.0000066 (\$/lit) & 0.00018 (\$/lit) \\
\hline 17 & Oil & 0 & 0 \\
\hline \multirow[t]{3}{*}{23} & Gas & 0.0000067 (\$/lit) & $0.000054\left(\$ / \mathrm{m}^{3}\right)$ \\
\hline & Oil & 0.0000064 (\$/lit) & 0.000052 (\$/lit) \\
\hline & Gasoil & 0.000013 (\$/lit) & 0.0001 (\$/lit) \\
\hline 30 & Oil & 0.0000083 (\$/lit) & 0.00013 (\$/lit) \\
\hline \multirow[t]{2}{*}{31} & Oil & 0.0000088 (\$/lit) & 0.00013 (\$/lit) \\
\hline & Gasoil & 0.0000057 (\$/lit) & 0.00026 (\$/lit) \\
\hline \multirow[t]{2}{*}{32} & Oil & 0.00000324 (\$/lit) & 0.000095 (\$/lit) \\
\hline & Gasoil & 0.00000647 (\$/lit) & 0.000187 (\$/lit) \\
\hline \multirow[t]{2}{*}{33} & Gas & $0.0000038\left(\$ / \mathrm{m}^{3}\right)$ & $0.00011\left(\$ / \mathrm{m}^{3}\right)$ \\
\hline & Gasoil & 0.0000076 (\$/lit) & 0.00022 (\$/lit) \\
\hline \multirow[t]{2}{*}{34} & Oil & 0.0000036 (\$/lit) & 0.00011 (\$/lit) \\
\hline & Gasoil & 0.0000072 (\$/lit) & 0.0002 (\$/lit) \\
\hline 36 & Oil & 0.0000039 (\$/lit) & 0.00011 (\$/lit) \\
\hline
\end{tabular}

system expansion planning, operation, and reliability. Due to their small size, widely known test systems (e.g., Garver's
Table 11 Fuel prices

\begin{tabular}{ll}
\hline Fuel & Price \\
\hline Gas & $0.00307\left(\$ / \mathrm{m}^{3}\right)$ \\
Oil & $0.00293(\$ /$ lit $)$ \\
Gasoil & $0.00586(\$ /$ lit $)$ \\
\hline
\end{tabular}

Table 12 Generating unit locations

\begin{tabular}{|c|c|c|c|}
\hline Bus & Size (MW) & No. of existing units & Max. number of units \\
\hline \multirow[t]{2}{*}{4} & 161 & 4 & 8 \\
\hline & 322 & 1 & 5 \\
\hline \multirow[t]{2}{*}{6} & 24 & 4 & 8 \\
\hline & 38.5 & 3 & 6 \\
\hline \multirow[t]{3}{*}{7} & 105 & 3 & 6 \\
\hline & 123.8 & 6 & 10 \\
\hline & 250 & 4 & 8 \\
\hline 10 & 156.5 & 4 & 8 \\
\hline \multirow[t]{2}{*}{11} & 102 & 3 & 6 \\
\hline & 116.2 & 6 & 10 \\
\hline 17 & 12.5 & 4 & 8 \\
\hline 23 & 82.5 & 3 & 6 \\
\hline 30 & 318 & 5 & 8 \\
\hline \multirow[t]{4}{*}{31} & 23.7 & 16 & 20 \\
\hline & 25 & 5 & 8 \\
\hline & 32 & 13 & 16 \\
\hline & 85 & 3 & 6 \\
\hline 32 & 159 & 12 & 16 \\
\hline \multirow[t]{2}{*}{33} & 100 & 2 & 5 \\
\hline & 128.5 & 4 & 8 \\
\hline \multirow[t]{2}{*}{34} & 166 & 5 & 8 \\
\hline & 332 & 1 & 5 \\
\hline \multirow[t]{2}{*}{36} & 263 & 3 & 6 \\
\hline & 526 & 3 & 6 \\
\hline
\end{tabular}

network and RBTS) may not adequately show the accuracy of proposed models. On the other hand, due to lack of information, larger test systems (e.g., RTS and Brazilian interconnections) may not be of use to authors of technical papers. Thus, the complete set of information exposed makes the presented test system highly useful to researchers in order to demonstrate robustness and effectiveness of proposed methodologies, evaluating present and future scenarios in the power system considering reliability and economic aspects. 


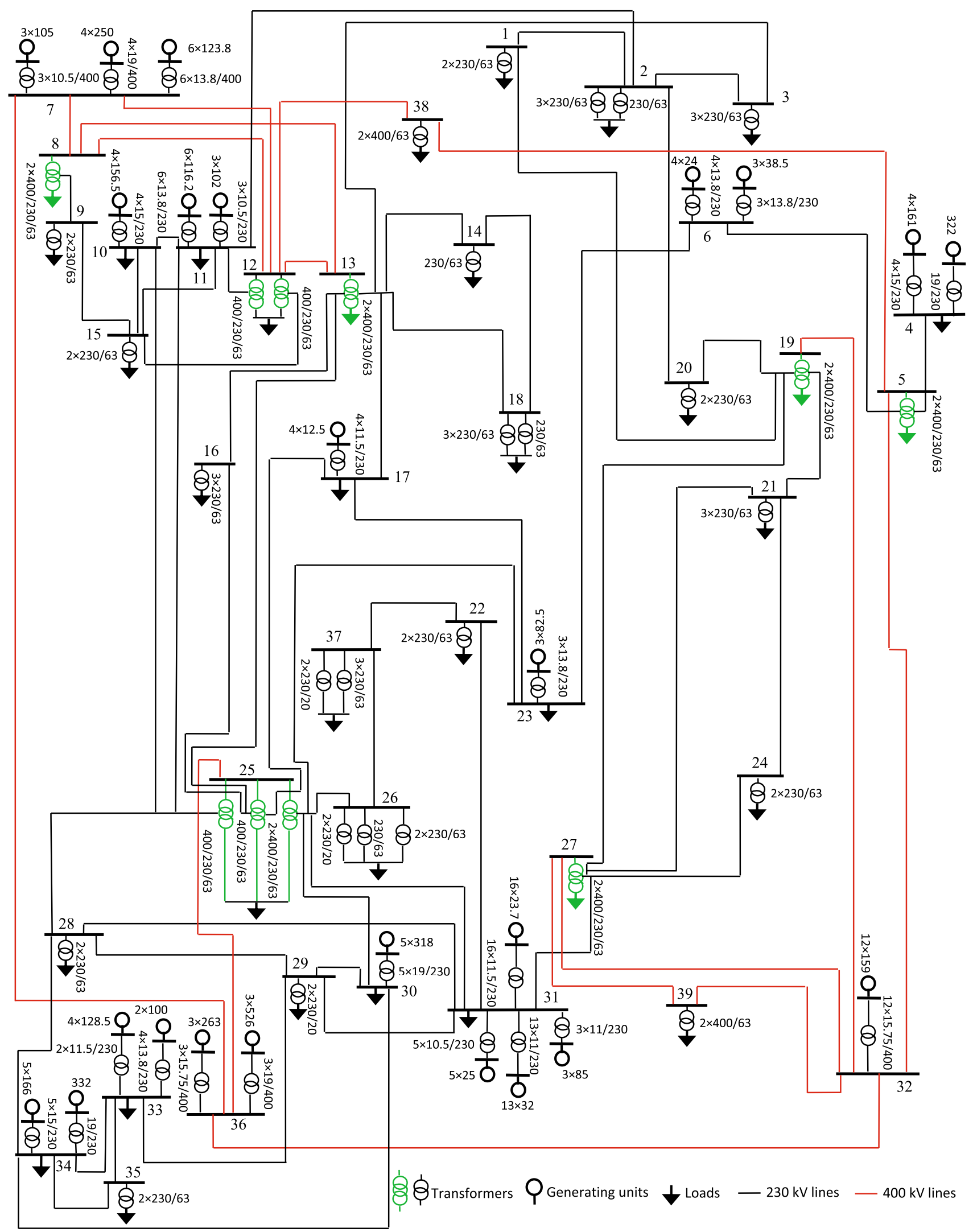

Fig. 1 RECT system 
Table 13 Generating unit reactive capability

\begin{tabular}{|c|c|c|c|c|c|}
\hline \multirow[t]{2}{*}{ Unit size (MW) } & \multicolumn{2}{|c|}{ MVAr } & \multirow[t]{2}{*}{ Unit size (MW) } & \multicolumn{2}{|c|}{ MVAr } \\
\hline & Min & Max & & Min & Max \\
\hline 12.5 & 0 & 8 & 123.8 & -25 & 60 \\
\hline 23.7 & 0 & 10 & 128.5 & 0 & 40 \\
\hline 24 & 0 & 10 & 156.5 & 0 & 25 \\
\hline 25 & 0 & 10 & 159 & 0 & 85 \\
\hline 32 & -10 & 15 & 161 & 0 & 85 \\
\hline 38.5 & -10 & 15 & 166 & -20 & 45 \\
\hline 82.5 & 0 & 35 & 250 & -40 & 130 \\
\hline 85 & -25 & 35 & 263 & 0 & 70 \\
\hline 100 & -25 & 30 & 318 & -25 & 90 \\
\hline 102 & 0 & 45 & 322 & -50 & 170 \\
\hline 105 & -30 & 50 & 332 & 0 & 90 \\
\hline 116.2 & -35 & 55 & 526 & -50 & 150 \\
\hline
\end{tabular}

Table 14 Voltage correction devices

\begin{tabular}{|c|c|c|c|c|c|c|c|}
\hline Bus & Device & $\begin{array}{l}\text { Amount } \\
\text { of } \\
\text { devices }\end{array}$ & $\begin{array}{l}\text { Capability of } \\
\text { each device (MVAr) }\end{array}$ & $\begin{array}{l}\text { Total } \\
\text { capability } \\
\text { (MVAr) }\end{array}$ & $\begin{array}{l}\text { Failure } \\
\text { rate }(\mathrm{h})\end{array}$ & MTTR (h) & FOD (h) \\
\hline 2 & Capacitor & 2 & 20 & 40 & 0.2 & 20 & 2 \\
\hline 3 & Capacitor & 2 & 20 & 40 & 0.22 & 18 & 1.8 \\
\hline 8 & Reactor & 4 & 25 & 100 & 0.16 & 24 & 0.7 \\
\hline 10 & Capacitor & 2 & 20 & 40 & 0.25 & 26 & 0.9 \\
\hline 12 & Reactor & 1 & 25 & 25 & 0.15 & 25 & 0.6 \\
\hline 16 & Capacitor & 2 & 10 & 20 & 0.22 & 32 & 1.5 \\
\hline 17 & Capacitor & 2 & 20 & 40 & 0.28 & 16 & 2.5 \\
\hline 19 & Reactor & 4 & 25 & 100 & 0.14 & 20 & 0.2 \\
\hline 21 & Capacitor & 2 & 20 & 40 & 0.18 & 28 & 0.7 \\
\hline 27 & Reactor & 2 & 25 & 50 & 0.14 & 30 & 1.4 \\
\hline 32 & Reactor & 3 & 50 & 150 & 0.17 & 35 & 0.8 \\
\hline 36 & Reactor & 2 & 50 & 100 & 0.16 & 22 & 0.3 \\
\hline
\end{tabular}

Table 15 Bus load data

\begin{tabular}{|c|c|c|c|c|c|c|c|}
\hline \multirow[t]{3}{*}{ Bus } & \multicolumn{2}{|l|}{ Load } & \multirow{2}{*}{\multicolumn{5}{|c|}{$\begin{array}{l}\text { Portion of load type from active bus load } \% \\
\text { Type }\end{array}$}} \\
\hline & \multirow[t]{2}{*}{$\overline{\mathrm{MW}}$} & \multirow[t]{2}{*}{$\overline{\text { MVAr }}$} & & & & & \\
\hline & & & 1 & 2 & 3 & 4 & 5 \\
\hline 1 & 138 & 34 & 50 & 28 & 2 & 9 & 11 \\
\hline 2 & 211 & 70 & 50 & 28 & 2 & 9 & 11 \\
\hline 3 & 339 & 250 & 50 & 28 & 2 & 9 & 11 \\
\hline 4 & 66 & 29 & 50 & 28 & 2 & 9 & 11 \\
\hline 5 & 125 & 57 & 50 & 28 & 2 & 9 & 11 \\
\hline 8 & 550 & 260 & 40 & 31 & 0.04 & 15 & 14 \\
\hline 9 & 190 & 68 & 40 & 31 & 0.04 & 15 & 14 \\
\hline 10 & 350 & 175 & 40 & 31 & 0.04 & 15 & 14 \\
\hline 11 & 170 & 96 & 40 & 31 & 0.04 & 15 & 14 \\
\hline 12 & 290 & 200 & 40 & 31 & 0.04 & 15 & 14 \\
\hline 13 & 620 & 350 & 40 & 31 & 0.04 & 15 & 14 \\
\hline 14 & 34 & 20 & 40 & 31 & 0.04 & 15 & 14 \\
\hline 15 & 290 & 154 & 40 & 31 & 0.04 & 15 & 14 \\
\hline
\end{tabular}


Table 15 continued

\begin{tabular}{|c|c|c|c|c|c|c|c|}
\hline \multirow[t]{3}{*}{ Bus } & \multicolumn{2}{|l|}{ Load } & \multirow{2}{*}{\multicolumn{5}{|c|}{$\begin{array}{l}\text { Portion of load type from active bus load } \% \\
\text { Type }\end{array}$}} \\
\hline & \multirow[t]{2}{*}{ MW } & \multirow[t]{2}{*}{ MVAr } & & & & & \\
\hline & & & 1 & 2 & 3 & 4 & 5 \\
\hline 16 & 354 & 210 & 39 & 20.5 & 5.5 & 30 & 5 \\
\hline 17 & 234 & 90 & 39 & 20.5 & 5.5 & 30 & 5 \\
\hline 18 & 480 & 220 & 50 & 28 & 2 & 9 & 11 \\
\hline 19 & 800 & 280 & 50 & 28 & 2 & 9 & 11 \\
\hline 20 & 210 & 116 & 50 & 28 & 2 & 9 & 11 \\
\hline 21 & 315 & 219 & 50 & 28 & 2 & 9 & 11 \\
\hline 22 & 168 & 96 & 40.5 & 28.5 & 0 & 4 & 27 \\
\hline 23 & 175 & 9 & 40.5 & 28.5 & 0 & 4 & 27 \\
\hline 24 & 206 & 116 & 45.5 & 15 & 5 & 24.5 & 10 \\
\hline 25 & 780 & 260 & 39 & 17 & 5.5 & 30 & 8.5 \\
\hline 26 & 746 & 390 & 40.5 & 28.5 & 0 & 4 & 27 \\
\hline 27 & 720 & 360 & 45.5 & 15 & 5 & 24.5 & 10 \\
\hline 28 & 268 & 114 & 42 & 16 & 2.5 & 24 & 15.5 \\
\hline 29 & 6 & 1 & 42 & 16 & 2.5 & 24 & 15.5 \\
\hline 30 & 230 & 92 & 42 & 16 & 2.5 & 24 & 15.5 \\
\hline 31 & 278 & 42 & 42 & 16 & 2.5 & 24 & 15.5 \\
\hline 33 & 128 & 42 & 37.5 & 13.5 & 15.5 & 26 & 7.5 \\
\hline 34 & 240 & 150 & 37.5 & 13.5 & 15.5 & 26 & 7.5 \\
\hline 35 & 64 & 20 & 37.5 & 13.5 & 15.5 & 26 & 7.5 \\
\hline 37 & 404 & 268 & 40.5 & 28.5 & 0 & 4 & 27 \\
\hline 38 & 250 & 120 & 40 & 31 & 0.04 & 15 & 14 \\
\hline 39 & 300 & 144 & 45.5 & 15 & 5 & 24.5 & 10 \\
\hline Total & & 10729 & & & 5122 & & \\
\hline
\end{tabular}

Table 16 Substation data: capacity and reliability

\begin{tabular}{|c|c|c|c|c|c|c|c|}
\hline Bus & $\begin{array}{l}\text { Voltage } \\
(\mathrm{kV})\end{array}$ & $\begin{array}{l}\text { Capacity of } \\
\text { transformers } \\
\text { (MVA) }\end{array}$ & $\begin{array}{l}\text { Failure rate } \\
\text { (1/year) }\end{array}$ & $\begin{array}{l}\text { Failure } \\
\text { duration (h) }\end{array}$ & $\begin{array}{l}\text { Repair rate } \\
\text { (1/year) }\end{array}$ & $\begin{array}{l}\text { Repair duration } \\
\text { (h) }\end{array}$ & $\begin{array}{l}\text { Age } \\
\text { (year) }\end{array}$ \\
\hline 1 & $230 / 63$ & $2 \times 160$ & 0.96 & 4.5 & 7 & 8 & 13 \\
\hline \multirow[t]{2}{*}{2} & \multirow[t]{2}{*}{$230 / 63$} & $1 \times 80$ & 0.9 & 9.5 & 11 & 9 & 42 \\
\hline & & $3 \times 90$ & 0.8 & 8 & 12 & 7 & 42 \\
\hline 3 & $230 / 63$ & $3 \times 180$ & 0.85 & 5.5 & 9 & 7 & 42 \\
\hline \multirow[t]{2}{*}{4} & $15 / 230$ & $4 \times 200$ & 0.75 & 6.5 & 15 & 6 & 20 \\
\hline & $19 / 230$ & $1 \times 350$ & 0.75 & 6.5 & 15 & 6 & 20 \\
\hline 5 & $400 / 230 / 63$ & $2 \times 500$ & 0.1 & 2 & 6.5 & 6.5 & 7 \\
\hline 6 & $13.8 / 230$ & $7 \times 40$ & 0.75 & 6.5 & 15 & 6 & 27 \\
\hline \multirow[t]{3}{*}{7} & $10.5 / 400$ & $3 \times 125$ & 0.1 & 2 & 6.5 & 6.5 & 14 \\
\hline & $13.8 / 400$ & $6 \times 154$ & 0.1 & 2 & 6.5 & 6.5 & 21 \\
\hline & $19 / 400$ & $4 \times 312.5$ & 0.1 & 2 & 6.5 & 6.5 & 23 \\
\hline 8 & $400 / 230 / 63$ & $2 \times 500$ & 0.05 & 3 & 5 & 8 & 37 \\
\hline 9 & $230 / 63$ & $2 \times 160$ & 0.96 & 4.5 & 7 & 8 & 14 \\
\hline 10 & $15 / 230$ & $4 \times 160$ & 0.7 & 5 & 6 & 10 & 45 \\
\hline \multirow[t]{2}{*}{11} & $13.8 / 230$ & $6 \times 140$ & 0.7 & 5 & 6 & 10 & 13 \\
\hline & $10.5 / 230$ & $3 \times 125$ & 0.7 & 5 & 6 & 10 & 13 \\
\hline
\end{tabular}


Table 16 continued

\begin{tabular}{|c|c|c|c|c|c|c|c|}
\hline Bus & $\begin{array}{l}\text { Voltage } \\
(\mathrm{kV})\end{array}$ & $\begin{array}{l}\text { Capacity of } \\
\text { transformers } \\
\text { (MVA) }\end{array}$ & $\begin{array}{l}\text { Failure rate } \\
\text { (1/year) }\end{array}$ & $\begin{array}{l}\text { Failure } \\
\text { duration (h) }\end{array}$ & $\begin{array}{l}\text { Repair rate } \\
\text { (1/year) }\end{array}$ & $\begin{array}{l}\text { Repair duration } \\
\text { (h) }\end{array}$ & $\begin{array}{l}\text { Age } \\
\text { (year) }\end{array}$ \\
\hline \multirow[t]{2}{*}{12} & \multirow[t]{2}{*}{$400 / 230 / 63$} & $1 \times 500$ & 0.05 & 3 & 5 & 8 & 13 \\
\hline & & $1 \times 500$ & 0.05 & 3 & 5 & 8 & 10 \\
\hline 13 & $400 / 230 / 63$ & $2 \times 500$ & 0.05 & 3 & 5 & 8 & 40 \\
\hline 14 & $230 / 63$ & $1 \times 160$ & 0.96 & 4.5 & 7 & 8 & 15 \\
\hline 15 & $230 / 63$ & $2 \times 250$ & 0.7 & 5 & 6 & 10 & 10 \\
\hline 16 & $230 / 63$ & $3 \times 180$ & 0.85 & 5.5 & 9 & 7 & 40 \\
\hline 17 & $11.5 / 230$ & $4 \times 35$ & 0.828 & 6.5 & 10 & 7.8 & 44 \\
\hline \multirow[t]{2}{*}{18} & \multirow[t]{2}{*}{$230 / 63$} & $3 \times 180$ & 0.85 & 5.5 & 9 & 7 & 26 \\
\hline & & $1 \times 180$ & 0.85 & 5.5 & 9 & 7 & 11 \\
\hline 19 & $400 / 230 / 63$ & $2 \times 500$ & 0.05 & 3 & 5 & 8 & 35 \\
\hline 20 & $230 / 63$ & $2 \times 160$ & 0.96 & 4.5 & 7 & 8 & 17 \\
\hline 21 & $230 / 63$ & $3 \times 180$ & 0.85 & 5.5 & 9 & 7 & 41 \\
\hline 22 & $230 / 63$ & $2 \times 180$ & 0.85 & 5.5 & 9 & 7 & 16 \\
\hline 23 & $13.8 / 230$ & $3 \times 100$ & 0.1 & 2 & 6.5 & 6.5 & 49 \\
\hline 24 & $230 / 63$ & $2 \times 160$ & 0.96 & 4.5 & 7 & 8 & 18 \\
\hline \multirow[t]{3}{*}{25} & \multirow[t]{3}{*}{$400 / 230 / 63$} & $2 \times 500$ & 0.05 & 3 & 5 & 8 & 34 \\
\hline & & $1 \times 500$ & 0.05 & 3 & 5 & 8 & 14 \\
\hline & & $1 \times 500$ & 0.05 & 3 & 5 & 8 & 7 \\
\hline \multirow[t]{3}{*}{26} & $230 / 63$ & $2 \times 180$ & 0.85 & 5.5 & 9 & 7 & 25 \\
\hline & $230 / 63$ & $1 \times 180$ & 0.85 & 5.5 & 9 & 7 & 10 \\
\hline & $230 / 20$ & $2 \times 90$ & 0.8 & 8 & 12 & 7 & 25 \\
\hline 27 & $400 / 230 / 63$ & $2 \times 500$ & 0.05 & 3 & 5 & 8 & 29 \\
\hline 28 & $230 / 63$ & $2 \times 160$ & 0.96 & 4.5 & 7 & 8 & 21 \\
\hline 29 & $230 / 20$ & $2 \times 40$ & 0.75 & 6.5 & 15 & 6 & 12 \\
\hline 30 & $19 / 230$ & $5 \times 350$ & 0.7 & 5 & 6 & 10 & 13 \\
\hline \multirow[t]{4}{*}{31} & $11.5 / 230$ & $16 \times 35$ & 0.7 & 5 & 6 & 10 & 33 \\
\hline & $10.5 / 230$ & $5 \times 35$ & 0.7 & 5 & 6 & 10 & 33 \\
\hline & $11 / 230$ & $13 \times 40$ & 0.7 & 5 & 6 & 10 & 33 \\
\hline & $11 / 230$ & $3 \times 110$ & 0.7 & 5 & 6 & 10 & 33 \\
\hline 32 & $15.75 / 400$ & $12 \times 200$ & 0.1 & 2 & 6.5 & 6.5 & 41 \\
\hline \multirow[t]{2}{*}{33} & $11.5 / 230$ & $2 \times 137.5$ & 0.7 & 5 & 6 & 10 & 38 \\
\hline & $13.8 / 230$ & $4 \times 126$ & 0.7 & 5 & 6 & 10 & 38 \\
\hline \multirow[t]{2}{*}{34} & $15 / 230$ & $5 \times 200$ & 0.7 & 5 & 6 & 10 & 21 \\
\hline & $19 / 230$ & $1 \times 350$ & 0.7 & 5 & 6 & 10 & 21 \\
\hline 35 & $230 / 63$ & $2 \times 40$ & 0.75 & 6.5 & 15 & 6 & 11 \\
\hline \multirow[t]{2}{*}{36} & $15.75 / 400$ & $3 \times 312.5$ & 0.1 & 2 & 6.5 & 6.5 & 31 \\
\hline & $19 / 400$ & $3 \times 550$ & 0.1 & 2 & 6.5 & 6.5 & 31 \\
\hline \multirow[t]{2}{*}{37} & $230 / 63$ & $3 \times 180$ & 0.85 & 5.5 & 9 & 7 & 21 \\
\hline & $230 / 20$ & $2 \times 90$ & 0.8 & 8 & 12 & 7 & 21 \\
\hline 38 & $400 / 63$ & $2 \times 200$ & 0.15 & 1 & 8 & 5 & 6 \\
\hline 39 & $400 / 63$ & $2 \times 200$ & 0.15 & 1 & 8 & 5 & 7 \\
\hline
\end{tabular}


Table 17 Costs of lost load and energy

\begin{tabular}{|c|c|c|c|c|c|}
\hline Load type & 1 & 2 & 3 & 4 & 5 \\
\hline VOLL (\$/MW) & 150 & 500 & 3500 & 1500 & 4500 \\
\hline Cost of ENS $(\$ / k W h)$ & 0.56 & 1.45 & 14 & 5.5 & 16.5 \\
\hline
\end{tabular}

Table 18 Load growth data (\%)

\begin{tabular}{|c|c|c|c|c|c|}
\hline \multirow[t]{2}{*}{ Bus } & \multicolumn{5}{|c|}{ Load type } \\
\hline & 1 & 2 & 3 & 4 & 5 \\
\hline 1 & 6.2 & 9.3 & 7.6 & 2 & 14 \\
\hline 2 & 6.2 & 9.3 & 7.6 & 2 & 14 \\
\hline 3 & 6.2 & 9.3 & 7.6 & 2 & 14 \\
\hline 4 & 6.2 & 9.3 & 7.6 & 2 & 14 \\
\hline 5 & 6.2 & 9.3 & 7.6 & 2 & 14 \\
\hline 8 & 3.9 & 7.9 & 17 & 4.8 & 13 \\
\hline 9 & 3.9 & 7.9 & 17 & 4.8 & 13 \\
\hline 10 & 3.9 & 7.9 & 17 & 4.8 & 13 \\
\hline 11 & 3.9 & 7.9 & 17 & 4.8 & 13 \\
\hline 12 & 3.9 & 7.9 & 17 & 4.8 & 13 \\
\hline 13 & 3.9 & 7.9 & 17 & 4.8 & 13 \\
\hline 14 & 3.9 & 7.9 & 17 & 4.8 & 13 \\
\hline 15 & 3.9 & 7.9 & 17 & 4.8 & 13 \\
\hline 16 & 9.3 & 10 & 9.5 & 8.3 & 8.6 \\
\hline 17 & 9.3 & 10 & 9.5 & 8.3 & 8.6 \\
\hline 18 & 6.2 & 9.3 & 7.6 & 2 & 14 \\
\hline 19 & 6.2 & 9.3 & 7.6 & 2 & 14 \\
\hline 20 & 6.2 & 9.3 & 7.6 & 2 & 14 \\
\hline 21 & 6.2 & 9.3 & 7.6 & 2 & 14 \\
\hline 22 & 3.2 & 18 & 0 & 6.6 & 6 \\
\hline 23 & 3.2 & 18 & 0 & 6.6 & 6 \\
\hline 24 & 6.1 & 8.3 & 14 & 5.8 & 7.2 \\
\hline 25 & 9.3 & 10 & 9.5 & 8.3 & 8.6 \\
\hline 26 & 3.2 & 18 & 0 & 6.6 & 6 \\
\hline 27 & 6.1 & 8.3 & 14 & 5.8 & 7.2 \\
\hline 28 & 6.4 & 2.8 & 4.8 & 7 & 7.1 \\
\hline 29 & 6.4 & 2.8 & 4.8 & 7 & 7.1 \\
\hline 30 & 6.4 & 2.8 & 4.8 & 7 & 7.1 \\
\hline 31 & 6.4 & 2.8 & 4.8 & 7 & 7.1 \\
\hline 33 & 11 & 15 & 5.4 & 10 & 8 \\
\hline 34 & 11 & 15 & 5.4 & 10 & 8 \\
\hline 35 & 11 & 15 & 5.4 & 10 & 8 \\
\hline 37 & 3.2 & 18 & 0 & 6.6 & 6 \\
\hline 38 & 3.9 & 7.9 & 17 & 4.8 & 13 \\
\hline 39 & 6.1 & 8.3 & 14 & 5.8 & 7.2 \\
\hline
\end{tabular}


Table 19 Transmission line data

\begin{tabular}{|c|c|c|c|c|c|c|c|c|c|c|}
\hline Corr & $\begin{array}{l}\text { Length } \\
(\mathrm{km})\end{array}$ & $\begin{array}{l}\text { Number of } \\
\text { circuits }\end{array}$ & $\begin{array}{l}\text { Number of } \\
\text { bundled } \\
\text { conductors }\end{array}$ & Type & $\begin{array}{l}\text { Voltage } \\
(\mathrm{kV})\end{array}$ & $\begin{array}{l}\text { Failure rate } \\
\text { (1/year) }\end{array}$ & $\begin{array}{l}\text { Failure } \\
\text { duration (h) }\end{array}$ & $\begin{array}{l}\text { Repair rate } \\
\text { (1/year) }\end{array}$ & $\begin{array}{l}\text { Repair } \\
\text { duration (h) }\end{array}$ & $\begin{array}{l}\text { Age } \\
\text { (year) }\end{array}$ \\
\hline $1-2$ & 9.5 & 1 & 2 & 1 & 230 & 0.15 & 4 & 0.3 & 11 & 13 \\
\hline $1-19$ & 21.5 & 1 & 2 & 1 & 230 & 0.3 & 4 & 0.33 & 11 & 13 \\
\hline $2-3$ & 17 & 1 & 2 & 1 & 230 & 0.26 & 4 & 0.33 & 11 & 40 \\
\hline $2-11$ & 55 & 1 & 2 & 1 & 230 & 0.85 & 6 & 0.4 & 15 & 19 \\
\hline $2-20$ & 15 & 1 & 2 & 1 & 230 & 0.23 & 4 & 0.32 & 11 & 18 \\
\hline $3-13$ & 30 & 1 & 2 & 1 & 230 & 0.46 & 4 & 0.35 & 11 & 19 \\
\hline $4-5$ & 20 & 1 & 1 & 1 & 230 & 0.3 & 3 & 0.33 & 10 & 10 \\
\hline $5-6$ & 9 & 1 & 1 & 1 & 230 & 0.14 & 3 & 0.31 & 10 & 11 \\
\hline $5-32$ & 75 & 2 & 3 & 3 & 400 & 0.7 & 11 & 0.52 & 35 & 9 \\
\hline $5-38$ & 50 & 2 & 3 & 3 & 400 & 0.47 & 9 & 0.47 & 25 & 8 \\
\hline $6-23$ & 53 & 2 & 1 & 1 & 230 & 0.8 & 5 & 0.4 & 12 & 30 \\
\hline $7-8$ & 25 & 2 & 3 & 3 & 400 & 0.23 & 6 & 0.43 & 15 & 26 \\
\hline $7-12$ & 110 & 2 & 3 & 3 & 400 & 1.03 & 13 & 0.59 & 40 & 7 \\
\hline $7-36$ & 110 & 2 & 3 & 3 & 400 & 1.02 & 13 & 0.58 & 40 & 16 \\
\hline $8-9$ & 33 & 1 & 1 & 2 & 230 & 0.5 & 3 & 0.36 & 10 & 14 \\
\hline $8-12$ & 82 & 1 & 3 & 3 & 400 & 0.77 & 11 & 0.53 & 35 & 14 \\
\hline $8-13$ & 101 & 1 & 3 & 3 & 400 & 0.95 & 11 & 0.57 & 35 & 16 \\
\hline $9-15$ & 23 & 1 & 1 & 2 & 230 & 0.35 & 3 & 0.34 & 10 & 14 \\
\hline $10-11$ & 3 & 2 & 2 & 1 & 230 & 0.05 & 4 & 0.3 & 11 & 20 \\
\hline $10-15$ & 18 & 1 & 1 & 2 & 230 & 0.27 & 3 & 0.33 & 10 & 21 \\
\hline $10-25$ & 31 & 1 & 2 & 1 & 230 & 0.48 & 4 & 0.35 & 11 & 40 \\
\hline $11-12$ & 18 & 2 & 2 & 1 & 230 & 0.27 & 4 & 0.33 & 11 & 13 \\
\hline $11-15$ & 17 & 1 & 2 & 1 & 230 & 0.26 & 4 & 0.33 & 11 & 8 \\
\hline $11-25$ & 28 & 1 & 1 & 1 & 230 & 0.43 & 3 & 0.35 & 10 & 49 \\
\hline $12-13$ & 17 & 2 & 2 & 3 & 400 & 0.26 & 4 & 0.33 & 11 & 14 \\
\hline $12-15$ & 7 & 1 & 2 & 1 & 230 & 0.11 & 4 & 0.3 & 11 & 8 \\
\hline $12-38$ & 25 & 2 & 3 & 3 & 400 & 0.23 & 6 & 0.43 & 15 & 9 \\
\hline $13-14$ & 10 & 1 & 2 & 2 & 230 & 0.15 & 4 & 0.31 & 11 & 8 \\
\hline $13-16$ & 11 & 1 & 2 & 1 & 230 & 0.17 & 4 & 0.31 & 11 & 40 \\
\hline $13-17$ & 8 & 2 & 2 & 1 & 230 & 0.12 & 4 & 0.31 & 11 & 40 \\
\hline $13-18$ & 14 & 1 & 2 & 2 & 230 & 0.21 & 4 & 0.32 & 11 & 15 \\
\hline $13-25$ & 24 & 1 & 2 & 1 & 230 & 0.37 & 4 & 0.34 & 11 & 40 \\
\hline $14-18$ & 7 & 1 & 2 & 2 & 230 & 0.11 & 4 & 0.33 & 11 & 8 \\
\hline $16-25$ & 13 & 1 & 2 & 1 & 230 & 0.2 & 4 & 0.32 & 11 & 40 \\
\hline $17-23$ & 15 & 1 & 1 & 1 & 230 & 0.23 & 4 & 0.32 & 11 & 7 \\
\hline $17-25$ & 14 & 2 & 1 & 1 & 230 & 0.21 & 3 & 0.32 & 10 & 10 \\
\hline 19-20 & 9 & 1 & 2 & 1 & 230 & 0.13 & 4 & 0.31 & 11 & 18 \\
\hline $19-21$ & 8 & 1 & 2 & 1 & 230 & 0.12 & 4 & 0.12 & 11 & 41 \\
\hline $19-27$ & 32 & 1 & 2 & 1 & 230 & 0.49 & 4 & 0.36 & 11 & 18 \\
\hline $19-32$ & 62 & 2 & 3 & 3 & 400 & 0.58 & 9 & 0.5 & 25 & 12 \\
\hline $21-27$ & 27 & 1 & 2 & 1 & 230 & 0.42 & 4 & 0.35 & 11 & 16 \\
\hline $21-24$ & 9 & 1 & 1 & 1 & 230 & 0.13 & 4 & 0.31 & 11 & 1 \\
\hline $22-31$ & 19 & 1 & 1 & 2 & 230 & 0.29 & 3 & 0.33 & 10 & 17 \\
\hline $22-37$ & 4.5 & - & - & 4 & 230 & 0 & 0 & 0.2 & 16 & 11 \\
\hline $23-25$ & 15 & 2 & 1 & 1 & 230 & 0.23 & 3 & 0.32 & 10 & 49 \\
\hline $24-27$ & 20 & 2 & 2 & 1 & 230 & 0.31 & 4 & 0.33 & 11 & 17 \\
\hline
\end{tabular}


Table 19 continued

\begin{tabular}{|c|c|c|c|c|c|c|c|c|c|c|}
\hline Corr & $\begin{array}{l}\text { Length } \\
(\mathrm{km})\end{array}$ & $\begin{array}{l}\text { Number of } \\
\text { circuits }\end{array}$ & $\begin{array}{l}\text { Number of } \\
\text { bundled } \\
\text { conductors }\end{array}$ & Type & $\begin{array}{l}\text { Voltage } \\
(\mathrm{kV})\end{array}$ & $\begin{array}{l}\text { Failure rate } \\
\text { (1/year) }\end{array}$ & $\begin{array}{l}\text { Failure } \\
\text { duration (h) }\end{array}$ & $\begin{array}{l}\text { Repair rate } \\
\text { (1/year) }\end{array}$ & $\begin{array}{l}\text { Repair } \\
\text { duration (h) }\end{array}$ & $\begin{array}{l}\text { Age } \\
\text { (year) }\end{array}$ \\
\hline $25-26$ & 6 & 2 & 2 & 2 & 230 & 0.09 & 4 & 0.3 & 11 & 29 \\
\hline $25-28$ & 11.5 & 1 & 1 & 2 & 230 & 0.18 & 3 & 0.31 & 10 & 11 \\
\hline $25-30$ & 40 & 2 & 2 & 2 & 230 & 0.62 & 6 & 0.37 & 15 & 7 \\
\hline $25-31$ & 15 & 1 & 1 & 2 & 230 & 0.23 & 3 & 0.32 & 10 & 11 \\
\hline $25-36$ & 46 & 2 & 3 & 3 & 400 & 0.43 & 9 & 0.47 & 25 & 34 \\
\hline 26-37 & 12 & - & - & 4 & 230 & 0 & 0 & 0.25 & 16 & 11 \\
\hline $27-31$ & 17 & 2 & 1 & 1 & 230 & 0.26 & 3 & 0.33 & 10 & 29 \\
\hline $27-32$ & 28 & 1 & 2 & 3 & 400 & 0.26 & 5 & 0.43 & 12 & 15 \\
\hline $27-39$ & 18 & 1 & 2 & 3 & 400 & 0.17 & 5 & 0.41 & 12 & 7 \\
\hline $28-29$ & 18 & 1 & 1 & 1 & 230 & 0.28 & 3 & 0.33 & 10 & 12 \\
\hline 28-31 & 21.5 & 1 & 1 & 2 & 230 & 0.33 & 3 & 0.33 & 10 & 11 \\
\hline $28-34$ & 120 & 1 & 1 & 1 & 230 & 1.85 & 8 & 0.54 & 20 & 4 \\
\hline 29-30 & 13 & 1 & 1 & 1 & 230 & 0.2 & 3 & 0.32 & 10 & 12 \\
\hline 29-31 & 38 & 1 & 1 & 1 & 230 & 0.58 & 5 & 0.37 & 12 & 12 \\
\hline 29-33 & 115 & 1 & 1 & 1 & 230 & 1.77 & 7 & 0.53 & 18 & 12 \\
\hline $30-34$ & 145 & 1 & 1 & 1 & 230 & 2.23 & 8 & 0.6 & 20 & 12 \\
\hline $32-36$ & 100 & 2 & 3 & 3 & 400 & 0.93 & 10 & 0.56 & 309 & 34 \\
\hline $32-39$ & 18 & 1 & 2 & 3 & 400 & 0.17 & 5 & 0.41 & 12 & 7 \\
\hline $33-34$ & 20 & 3 & 2 & 1 & 230 & 0.31 & 4 & 0.33 & 11 & 22 \\
\hline $33-35$ & 40 & 2 & 1 & 1 & 230 & 0.61 & 5 & 0.37 & 12 & 9 \\
\hline 34-35 & 30 & 1 & 1 & 1 & 230 & 0.46 & 4 & 0.35 & 11 & 2 \\
\hline
\end{tabular}

Table 20 Corridor length

\begin{tabular}{|c|c|c|c|c|c|c|c|c|c|c|c|}
\hline Corr & Length (km) & Corr & Length (km) & Corr & Length (km) & Corr & Length (km) & Corr & Length (km) & Corr & Length $(\mathrm{km})$ \\
\hline $1-3$ & 22 & $2-14$ & 15 & $6-13$ & 55 & $10-12$ & 19 & $13-39$ & 12 & $25-29$ & 30 \\
\hline $1-4$ & 70 & $2-18$ & 14 & $6-14$ & 8 & $10-13$ & 25 & $14-17$ & 14 & $25-37$ & 14 \\
\hline $1-5$ & 65 & $2-19$ & 10 & $6-18$ & 10 & $10-16$ & 25 & $14-19$ & 18 & $26-27$ & 10 \\
\hline $1-6$ & 12 & $2-21$ & 10 & $6-19$ & 6 & $10-26$ & 42 & $14-20$ & 13 & $26-28$ & 8 \\
\hline $1-8$ & 100 & $3-4$ & 55 & $6-20$ & 7 & $10-28$ & 40 & $14-37$ & 14 & $26-29$ & 12 \\
\hline $1-9$ & 60 & $3-5$ & 65 & $7-13$ & 127 & $11-13$ & 26 & $14-38$ & 6 & $26-30$ & 15 \\
\hline $1-10$ & 52 & $3-6$ & 7 & $7-23$ & 60 & $11-14$ & 33 & $15-16$ & 25 & $26-33$ & 120 \\
\hline $1-11$ & 50 & $3-8$ & 41 & $7-25$ & 47 & $11-16$ & 25 & $15-25$ & 15 & $27-28$ & 25 \\
\hline $1-12$ & 32 & $3-10$ & 42 & $8-10$ & 65 & $11-26$ & 40 & $15-28$ & 21 & $27-29$ & 60 \\
\hline $1-13$ & 21 & $3-11$ & 40 & $8-11$ & 66.5 & $11-28$ & 40 & $16-18$ & 14.5 & $27-30$ & 50 \\
\hline $1-14$ & 9 & $3-12$ & 35 & $8-15$ & 45 & $12-14$ & 22 & $16-28$ & 16 & $27-37$ & 11 \\
\hline $1-18$ & 10 & $3-18$ & 14 & $8-23$ & 30 & $12-16$ & 14 & $17-38$ & 14 & $28-30$ & 35 \\
\hline $1-20$ & 15 & $3-19$ & 8 & $8-25$ & 60 & $12-18$ & 24 & $18-20$ & 7 & $28-33$ & 110 \\
\hline $1-21$ & 20 & $4-6$ & 7 & $8-26$ & 10 & $12-19$ & 35 & $18-37$ & 10 & $28-37$ & 14 \\
\hline $1-37$ & 25 & 4-19 & 58 & $8-28$ & 65 & $12-20$ & 28 & $19-23$ & 16 & $29-34$ & 120 \\
\hline
\end{tabular}


Table 20 continued

\begin{tabular}{lcllllllllll}
\hline Corr & Length $(\mathrm{km})$ & Corr & Length $(\mathrm{km})$ & Corr & Length $(\mathrm{km})$ & Corr & Length $(\mathrm{km})$ & Corr & Length $(\mathrm{km})$ & Corr & Length $(\mathrm{km})$ \\
\hline $2-4$ & 65 & $4-21$ & 65 & $9-10$ & 45 & $12-25$ & 40 & $19-26$ & 24 & $29-35$ & 140 \\
$2-5$ & 75 & $4-24$ & 70 & $9-11$ & 47 & $12-28$ & 32 & $19-38$ & 18 & $30-31$ & 28 \\
$2-6$ & 7 & $5-13$ & 80 & $9-12$ & 57 & $12-37$ & 31 & $20-21$ & 9 & $30-33$ & 123 \\
$2-8$ & 105 & $5-19$ & 30 & $9-13$ & 70 & $12-39$ & 20 & $21-26$ & 17 & $30-35$ & 147 \\
$2-9$ & 65 & $5-21$ & 40 & $9-16$ & 55 & $13-19$ & 35 & $22-26$ & 7 & $31-33$ & 150 \\
$2-10$ & 55 & $5-24$ & 45 & $9-25$ & 41 & $13-20$ & 18 & $22-28$ & 16 & $31-35$ & 180 \\
$2-12$ & 35 & $6-11$ & 65 & $9-26$ & 66 & $13-28$ & 22 & $23-27$ & 6 & $31-37$ & 20 \\
$2-13$ & 24 & $6-12$ & 45 & $9-28$ & 47 & $13-37$ & 21 & $24-26$ & 14 & $36-39$ & 70 \\
\hline
\end{tabular}

Table 21 Impedance, rating data $\left(S_{b}=100 \mathrm{MVA}\right)$ and construction costs (Haddadian et al. 2011)

\begin{tabular}{|c|c|c|c|c|c|c|c|c|}
\hline Volta & ) Type & $\begin{array}{l}\text { Number of } \\
\text { bundled conduc- } \\
\text { tors }\end{array}$ & $\begin{array}{l}\text { Rating } \\
\text { (P.U.) }\end{array}$ & $\begin{array}{l}\text { Resistance } \\
(\text { P.U./km) } \\
\times 10 \mathrm{e}-4\end{array}$ & $\begin{array}{l}\text { Reactance } \\
(\text { P.U./km) } \\
\times 10 \mathrm{e}-4\end{array}$ & $\begin{array}{l}\text { Susceptance } \\
(\mathrm{P} . \mathrm{U} . / \mathrm{km}) \\
\times 10 \mathrm{e}-4\end{array}$ & $\begin{array}{l}\text { Constant } \\
\text { cost }(\$) \times 10 \mathrm{e} 3\end{array}$ & $\begin{array}{l}\text { Variable } \\
\text { cost }(\$) \times 10 \mathrm{e} 3\end{array}$ \\
\hline \multirow{5}{*}{230} & \multirow[t]{2}{*}{ CANARY } & 1 & 4 & 1.22 & 3.85 & 19 & 500 & 42 \\
\hline & & 2 & 8 & 0.61 & 2.84 & 24 & 500 & 43 \\
\hline & \multicolumn{2}{|c|}{ CARDINAL 1} & 4.5 & 1.16 & 3.85 & 19 & 500 & 45 \\
\hline & & 2 & 8.5 & 0.58 & 2.82 & 24.5 & 500 & 46 \\
\hline & Cable & - & 3.45 & 1 & 16 & 3200 & 200 & 60 \\
\hline \multirow[t]{3}{*}{400} & \multirow[t]{3}{*}{ CERLEW } & 1 & 7.5 & 0.35 & 1.24 & 58 & 1600 & 85 \\
\hline & & 2 & 15 & 0.175 & 0.97 & 74 & 1600 & 86.5 \\
\hline & & 3 & 25 & 0.115 & 0.86 & 83 & 1600 & 87 \\
\hline
\end{tabular}

\section{Appendix: Capacity Outage Probability Cal- culation}

Several indices can be calculated in transmission systems in order to asses the operation reliability. Hereby, the capacity outage probability for the RECT system is presented in
Table 22, enabling the calculation of the main reliability criteria (e.g., loss of load probability (LOLP) and loss of load expectation (LOLE)) in further studies. Table 22 includes the capacity outage probabilities (COP) in the range of 0 $100 \mathrm{MW}$. 
Table 22 Capacity outage probability table (0-100 MW)

\begin{tabular}{|c|c|c|c|c|c|c|c|}
\hline Cap. out (MW) & Cap. in (MW) & Ind. prob. & Cum. prob. & Cap. out (MW) & Cap. in (MW) & Ind. prob. & Cum. prob. \\
\hline 0 & $14,078.2$ & $1.01 \mathrm{E}-05$ & 1.0000000000 & $79.7=32+23.7+24$ & $13,998.5$ & $5.86 \mathrm{E}-06$ & 0.9992957224 \\
\hline 12.5 & $14,065.7$ & $1.50 \mathrm{E}-06$ & 0.9999899350 & $80=2 \times 24+32$ & $13,998.2$ & $9.97 \mathrm{E}-07$ & 0.9992898640 \\
\hline 23.7 & $14,054.5$ & $3.30 \mathrm{E}-05$ & 0.9999884315 & $80.7=2 \times 12.5+23.7+32$ & $13,997.5$ & $7.35 \mathrm{E}-07$ & 0.9992888668 \\
\hline 24 & $14,054.2$ & $3.17 \mathrm{E}-06$ & 0.9999554465 & $81=2 \times 12.5+32+24$ & $13,997.2$ & $7.06 \mathrm{E}-08$ & 0.9992881319 \\
\hline 25 & $14,053.2$ & $1.03 \mathrm{E}-05$ & 0.9999451385 & $82=2 \times 25+32$ & $13,996.2$ & $1.12 \mathrm{E}-05$ & 0.9992880612 \\
\hline $25=2 \times 12.5$ & $14,053.2$ & $8.42 \mathrm{E}-08$ & 0.9999348305 & $82=4 \times 12.5+32$ & $13,996.2$ & $5.21 \mathrm{E}-11$ & 0.9992768182 \\
\hline 32 & $14,046.2$ & $3.30 \mathrm{E}-05$ & 0.9999347463 & 82.5 & $13,995.7$ & $2.63 \mathrm{E}-06$ & 0.9992768182 \\
\hline $36.2=12.5+23.7$ & 14,042 & $1.61 \mathrm{E}-05$ & 0.9999017613 & $83.6=3 \times 23.7+12.5$ & $13,994.6$ & $6.20 \mathrm{E}-06$ & 0.9992741925 \\
\hline $36.5=12.5+24$ & $14,041.7$ & $4.74 \mathrm{E}-07$ & 0.9998856143 & $83.9=12.5+2 \times 23.7+24$ & $13,994.3$ & $2.38 \mathrm{E}-06$ & 0.9992679915 \\
\hline $37.5=3 \times 12.5$ & $14,040.7$ & $2.10 \mathrm{E}-09$ & 0.9998851407 & $84.2=12.5+23.7+2 \times 24$ & 13,994 & $9.94 \mathrm{E}-07$ & 0.9992656074 \\
\hline $37.5=12.5+25$ & $14,040.7$ & $1.54 \mathrm{E}-06$ & 0.9998851386 & $84.5=3 \times 24+12.5$ & $13,993.7$ & $2.94 \mathrm{E}-09$ & 0.9992646132 \\
\hline 38.5 & $14,039.7$ & $2.34 \mathrm{E}-06$ & 0.9998835989 & $84.9=12.5+2 \times 23.7+25$ & $13,993.3$ & $7.75 \mathrm{E}-06$ & 0.9992646103 \\
\hline $44.5=12.5+32$ & $14,033.7$ & $4.00 \mathrm{E}-06$ & 0.9998812526 & $84.9=2 \times 23.7+3 \times 12.5$ & $13,993.3$ & $1.06 \mathrm{E}-08$ & 0.9992568591 \\
\hline $47.4=2 \times 23.7$ & $14,030.8$ & $5.07 \mathrm{E}-05$ & 0.9998772529 & 85 & $13,993.2$ & $5.22 \mathrm{E}-07$ & 0.9992568486 \\
\hline $47.7=23.7+24$ & $14,030.5$ & $1.04 \mathrm{E}-05$ & 0.9998265839 & $85.2=3 \times 12.5+23.7+24$ & 13,993 & $2.16 \mathrm{E}-09$ & 0.9992563264 \\
\hline $48=2 \times 24$ & $14,030.2$ & $3.75 \mathrm{E}-07$ & 0.9998161939 & $85.5=2 \times 24+3 \times 12.5$ & $13,992.7$ & $6.70 \mathrm{E}-11$ & 0.9992563242 \\
\hline $48.7=23.7+25$ & $14,029.5$ & $3.38 \mathrm{E}-05$ & 0.9998158561 & $85.5=12.5+25+2 \times 24$ & $13,992.7$ & $5.73 \mathrm{E}-08$ & 0.9992563242 \\
\hline $48.7=2 \times 12.5+23.7$ & $14,029.5$ & $2.76 \mathrm{E}-07$ & 0.9997820771 & $85.9=2 \times 23.7+38.5$ & $13,992.3$ & $1.18 \mathrm{E}-05$ & 0.9992562669 \\
\hline $49=24+25$ & $14,029.2$ & $3.25 \mathrm{E}-06$ & 0.9997818011 & $86.2=38.5+23.7+24$ & 13,992 & $2.42 \mathrm{E}-06$ & 0.9992444729 \\
\hline $49=2 \times 12.5+24$ & $14,029.2$ & $2.65 \mathrm{E}-08$ & 0.9997785542 & $86.2=12.5+23.7+2 \times 25$ & 13,992 & $1.42 \mathrm{E}-06$ & 0.9992420545 \\
\hline $50=4 \times 12.5$ & $14,028.2$ & $1.96 \mathrm{E}-11$ & 0.9997785277 & $86.2=3 \times 12.5+23.7+25$ & 13,992 & $7.04 \mathrm{E}-09$ & 0.9992406306 \\
\hline $50=2 \times 25$ & $14,028.2$ & $4.22 \mathrm{E}-06$ & 0.9997785277 & $86.5=2 \times 24+38.5$ & $13,991.7$ & $8.72 \mathrm{E}-08$ & 0.9992406236 \\
\hline $50=2 \times 12.5+25$ & $14,028.2$ & $8.63 \mathrm{E}-08$ & 0.9997743053 & $86.5=12.5+2 \times 25+24$ & $13,991.7$ & $1.99 \mathrm{E}-07$ & 0.9992405364 \\
\hline $51=12.5+38.5$ & $14,027.2$ & $3.50 \mathrm{E}-07$ & 0.9997742191 & $86.5=3 \times 12.5+25+24$ & $13,991.7$ & $6.76 \mathrm{E}-10$ & 0.9992403377 \\
\hline $55.7=23.7+32$ & $14,022.5$ & $8.78 \mathrm{E}-05$ & 0.9997738692 & $87.2=2 \times 12.5+23.7+38.5$ & 13,991 & $6.42 \mathrm{E}-08$ & 0.9992403370 \\
\hline $56=24+32$ & $14,022.2$ & $8.44 \mathrm{E}-06$ & 0.9996860432 & $87.5=2 \times 25+3 \times 12.5$ & $13,990.7$ & $8.80 \mathrm{E}-10$ & 0.9992402728 \\
\hline $57=25+32$ & $14,021.2$ & $2.74 \mathrm{E}-05$ & 0.9996776014 & $87.5=3 \times 25+12.5$ & $13,990.7$ & $1.29 \mathrm{E}-07$ & 0.9992402719 \\
\hline $57=2 \times 12.5+32$ & $14,021.2$ & $2.24 \mathrm{E}-07$ & 0.9996501554 & $87.5=2 \times 12.5+38.5+24$ & $13,990.7$ & $6.17 \mathrm{E}-09$ & 0.9992401427 \\
\hline $59.9=12.5+2 \times 23.7$ & $14,018.3$ & $7.57 \mathrm{E}-06$ & 0.9996499312 & $87.7=2 \times 32+23.7$ & $13,990.5$ & $1.08 \mathrm{E}-04$ & 0.9992401365 \\
\hline $60.2=12.5+23.7+24$ & 14,018 & $1.55 \mathrm{E}-06$ & 0.9996423624 & $88=2 \times 32+24$ & $13,990.2$ & $1.04 \mathrm{E}-05$ & 0.9991322065 \\
\hline $60.5=12.5+2 \times 24$ & $14,017.7$ & $5.59 \mathrm{E}-08$ & 0.9996408104 & $88.5=2 \times 25+38.5$ & $13,989.7$ & $9.83 \mathrm{E}-07$ & 0.9991218325 \\
\hline $61.2=3 \times 12.5+23.7$ & 14,017 & $6.87 \mathrm{E}-09$ & 0.9996407545 & $89=2 \times 32+25$ & $13,989.2$ & $3.37 \mathrm{E}-05$ & 0.9991208497 \\
\hline $61.2=12.5+23.7+25$ & 14,017 & $5.05 \mathrm{E}-06$ & 0.9996407476 & $89=2 \times 12.5+2 \times 32$ & $13,989.2$ & $2.76 \mathrm{E}-07$ & 0.9990871207 \\
\hline $61.5=3 \times 12.5+24$ & $14,016.7$ & $6.60 \mathrm{E}-10$ & 0.9996357017 & $89.5=2 \times 38.5+12.5$ & $13,988.7$ & $2.72 \mathrm{E}-08$ & 0.9990868451 \\
\hline $61.5=12.5+24+25$ & $14,016.7$ & $4.85 \mathrm{E}-07$ & 0.9996357010 & $91.9=12.5+2 \times 23.7+32$ & $13,986.3$ & $2.02 \mathrm{E}-05$ & 0.9990868180 \\
\hline $62.2=23.7+38.5$ & 14,016 & $7.68 \mathrm{E}-06$ & 0.9996352160 & $92.5=12.5+32+2 \times 24$ & $13,985.7$ & $1.49 \mathrm{E}-07$ & 0.9990666650 \\
\hline $62.5=24+38.5$ & $14,015.7$ & $7.38 \mathrm{E}-07$ & 0.9996275386 & $93.2=3 \times 12.5+23.7+32$ & 13,985 & $1.83 \mathrm{E}-08$ & 0.9990665160 \\
\hline $62.5=12.5+2 \times 25$ & $14,015.7$ & $6.31 \mathrm{E}-07$ & 0.9996268007 & $93.5=3 \times 12.5+32+24$ & 984.7 & $1.76 \mathrm{E}-09$ & 0.9990664977 \\
\hline $62.5=3 \times 12.5+25$ & 14015.7 & $2.15 \mathrm{E}-09$ & 0.9996261700 & $94.8=4 \times 23.7$ & 13983.4 & $2.76 \mathrm{E}-05$ & 0.9990664960 \\
\hline $63.5=25+38.5$ & $14,014.7$ & $2.40 \mathrm{E}-06$ & 0.9996261679 & $95=12.5+82.5$ & $13,983.2$ & $3.92 \mathrm{E}-07$ & 0.9990388630 \\
\hline $63.5=2 \times 12.5+38.5$ & $14,014.7$ & $6.53 \mathrm{E}-09$ & 0.9996237687 & $95.1=3 \times 23.7+24$ & $13,983.1$ & $1.31 \mathrm{E}-05$ & 0.9990384708 \\
\hline $64=2 \times 32$ & $14,014.2$ & $3.29 \mathrm{E}-05$ & 0.9996237622 & $95.4=2 \times 23.7+2 \times 24$ & $13,982.8$ & $1.89 \mathrm{E}-06$ & 0.9990253948 \\
\hline $68.2=12.5+23.7+32$ & 14,010 & $1.31 \mathrm{E}-05$ & 0.9995908272 & $95.7=3 \times 24+23.7$ & $13,982.5$ & $6.44 \mathrm{E}-08$ & 0.9990235095 \\
\hline $68.5=12.5+24+32$ & $14,009.7$ & $1.26 \mathrm{E}-06$ & 0.9995777082 & $96=4 \times 24$ & $13,982.2$ & $3.87 \mathrm{E}-10$ & 0.9990234451 \\
\hline $69.5=3 \times 12.5+32$ & $14,008.7$ & $5.88 \mathrm{E}-07$ & 0.9995764472 & $96=3 \times 32$ & $13,982.2$ & $2.47 \mathrm{E}-05$ & 0.9990234447 \\
\hline $70.5=32+38.5$ & $14,007.7$ & $6.24 \mathrm{E}-06$ & 0.9995758593 & $96.1=2 \times 12.5+3 \times 23.7$ & $13,982.1$ & $3.47 \mathrm{E}-07$ & 0.9989987107 \\
\hline
\end{tabular}


Table 22 continued

\begin{tabular}{|c|c|c|c|c|c|c|c|}
\hline Cap. out (MW) & Cap. in (MW) & Ind. prob. & Cum. prob. & Cap. out (MW) & Cap. in (MW) & Ind. prob. & Cum. prob. \\
\hline $71.1=3 \times 23.7$ & $14,007.1$ & $4.15 \mathrm{E}-05$ & 0.9995696214 & $96.1=3 \times 23.7+25$ & $13,982.1$ & $4.25 \mathrm{E}-05$ & 0.9989983633 \\
\hline $71.4=2 \times 23.7+24$ & $14,006.8$ & $1.60 \mathrm{E}-05$ & 0.9995281094 & $96.4=25+2 \times 23.7+24$ & $13,981.8$ & $1.63 \mathrm{E}-05$ & 0.9989558513 \\
\hline $71.7=23.7+2 \times 24$ & $14,006.5$ & $1.23 \mathrm{E}-06$ & 0.9995121494 & $96.4=2 \times 12.5+2 \times 23.7+24$ & $13,981.8$ & $1.47 \mathrm{E}-08$ & 0.9989395063 \\
\hline $72=3 \times 24$ & $14,006.2$ & $1.97 \mathrm{E}-08$ & 0.9995109221 & $96.7=25+23.7+2 \times 24$ & $13,981.5$ & $1.26 \mathrm{E}-06$ & 0.9989394916 \\
\hline $72.4=2 \times 23.7+25$ & $14,005.8$ & $5.19 \mathrm{E}-05$ & 0.9995109024 & $97=2 \times 12.5+3 \times 24$ & $13,981.2$ & $1.65 \mathrm{E}-10$ & 0.9989382347 \\
\hline $72.7=23.7+24+25$ & $14,005.5$ & $1.06 \mathrm{E}-05$ & 0.9994590124 & $97=3 \times 24+25$ & $13,981.2$ & $2.01 \mathrm{E}-08$ & 0.9989382345 \\
\hline $72.7=2 \times 12.5+23.7+24$ & $14,005.5$ & $8.69 \mathrm{E}-08$ & 0.9994483724 & $97.4=2 \times 23.7+4 \times 12.5$ & $13,980.8$ & $9.85 \mathrm{E}-11$ & 0.9989382144 \\
\hline $73=2 \times 24+25$ & $14,005.2$ & $3.84 \mathrm{E}-07$ & 0.9994482855 & $97.4=2 \times 23.7+2 \times 25$ & $13,980.8$ & $2.13 \mathrm{E}-05$ & 0.9989382144 \\
\hline $73=2 \times 12.5+2 \times 24$ & $14,005.2$ & $3.13 \mathrm{E}-09$ & 0.9994479020 & $97.4=2 \times 12.5+2 \times 23.7+25$ & $13,980.8$ & $4.34 \mathrm{E}-07$ & 0.9988947944 \\
\hline $73.7=23.7+2 \times 25$ & $14,004.5$ & $9.53 \mathrm{E}-06$ & 0.9994478989 & $97.5=12.5+85$ & $13,980.7$ & $7.80 \mathrm{E}-08$ & 0.998894360 \\
\hline $73.7=4 \times 12.5+23.7$ & $14,004.5$ & -11 & 0.9994383664 & $97.7=2 \times 25+23.7+24$ & .5 & $5 E-06$ & 0.998894282 \\
\hline $73.7=2 \times 12.5+23.7+25$ & $14,004.5$ & $2.63 \mathrm{E}-07$ & 0.9994383664 & $98=2 \times 24+4 \times 12.5$ & $13,980.2$ & $7.28 \mathrm{E}-13$ & 0.998889923 \\
\hline $74=24+2 \times 25$ & $14,004.2$ & $1.33 \mathrm{E}-06$ & 0.9994381037 & $98=2 \times 24+2 \times 25$ & $13,980.2$ & $1.57 \mathrm{E}-07$ & 0.998889923 \\
\hline $74=4 \times 12.5+24$ & $14,004.2$ & $6.17 \mathrm{E}-12$ & 0.9994367737 & $98.4=12.5+2 \times 23.7+38.5$ & $13,979.8$ & $1.76 \mathrm{E}-06$ & 0.998889766 \\
\hline $74=2 \times 12.5+24+25$ & $14,004.2$ & $2.72 \mathrm{E}-08$ & 0.9994367737 & $98.7=3 \times 25+23.7$ & $13,979.5$ & $2.83 \mathrm{E}-06$ & 0.998888004 \\
\hline $74.7=12.5+23.7+38.5$ & $14,003.5$ & $1.49 \mathrm{E}-09$ & 0.9994367456 & $99=3 \times 25+24$ & $13,979.2$ & $2.72 \mathrm{E}-07$ & 0.9988851705 \\
\hline $75=3 \times 25$ & $14,003.2$ & $8.65 \mathrm{E}-07$ & 0.9994367450 & $99=12.5+38.5+2 \times 24$ & $13,979.2$ & $1.30 \mathrm{E}-08$ & 0.9988848981 \\
\hline $75=2 \times 12.5+2 \times 25$ & $14,003.2$ & $3.53 \mathrm{E}-08$ & 0.9994358802 & $99=2 \times 12.5+2 \times 25+24$ & $13,979.2$ & $1.11 \mathrm{E}-08$ & 0.9988848851 \\
\hline $75=4 \times 12.5+25$ & $14,003.2$ & $1.60 \mathrm{E}-11$ & 0.9994358449 & $99.7=3 \times 12.5+23.7+38.5$ & $13,978.5$ & $1.60 \mathrm{E}-09$ & 0.9988848740 \\
\hline $75=12.5+24+38.5$ & $14,003.2$ & $1.10 \mathrm{E}-07$ & 0.9994358449 & 100 & $13,978.2$ & $1.28 \mathrm{E}-06$ & 0.9988848724 \\
\hline $76=3 \times 12.5+38.5$ & $14,002.2$ & $4.88 \mathrm{E}-10$ & 0.9994357347 & $100=4 \times 25$ & $13,978.2$ & $8.86 \mathrm{E}-08$ & 0.9988835875 \\
\hline $76.5=12.5+2 \times 32$ & $14,001.7$ & $4.92 \mathrm{E}-06$ & 0.9994357342 & $100=2 \times 12.5+3 \times 25$ & $13,978.2$ & $7.24 \mathrm{E}-09$ & 0.9988834989 \\
\hline $77=2 \times 38.5$ & $14,001.2$ & $1.82 \mathrm{E}-07$ & 0.9994308142 & $100=2 \times 25+4 \times 12.5$ & $13,978.2$ & $8.21 \mathrm{E}-12$ & 0.9988834917 \\
\hline $79.4=2 \times 23.7+32$ & $13,998.8$ & $1.35 \mathrm{E}-04$ & 0.9994306324 & $100=3 \times 12.5+38.5+24$ & $13,978.2$ & $1.54 \mathrm{E}-10$ & 0.9988834917 \\
\hline
\end{tabular}

\section{References}

Billinton, R. (1989). A reliability test system for educational purposesbasic data. IEEE Transactions on Power Systems, 4, 1238-1244.

Braga, A. S. D., \& Saraiva, J. T. (2005). A multiyear dynamic approach for transmission expansion planning and long-term marginal costs computation. IEEE Transactions on Power Systems, 20, 16311639 .

Choi, J., Mount, T. D., Thomas, R. J., \& Billinton, R. (2006). Probabilistic reliability criterion for planning transmission system expansions. IEE Proceedings-Generation, Transmission and Distribution, 153, 719-727.

Ekwue, A. O., \& Cory, B. J. (1984). Transmission system expansion planning by interactive methods. IEEE Transactions on Power Apparatus and Systems, PAS-103, 1583-1591.

Escobar, A. (2002). Planeamiento Dinamico de la expansion de sistemas de transmision usando algoritmos sistemas de transmision usando algoritmos, Ph.D. thesis, Universidad Technologica De Pereira.

Escobar, A. H., Gallego, R. A., \& Romero, R. (2004). Multistage and coordinated planning of the expansion of transmission systems. IEEE Transactions on Power Systems, 19, 735-734.

Fang, R., \& Hill, D. J. (2003). A new strategy for transmission expansion in competitive electricity markets. IEEE Transactions on Power Systems, 18, 374-380.

Garver, L. L. (1970). Transmission network estimation using linear programming. IEEE Transactions on Power Apparatus and Systems, PAS-89, 1688-1697.

Haddadian, H., Hosseini, S. H., Shayeghi, H., \& Shayanfar, H. A. (2011). Determination of optimum generation level in DTEP using a GA-based quadratic programming. Energy Conversion and Management, 52, 382-390.

IEEE Reliability Test System Task Force of the Application of Probability Methods Subcommittee. (1979). IEEE reliability test system. In IEEE Transactions on Power Apparatus Systems, PAS-98 (pp. 2047-2054)

Maghouli, P., Hosseini, S. H., Buygi, M. O., \& Shahidehpour, M. (2011). A scenario-based multi-objective model for multi-stage transmission expansion planning. IEEE Transactions on Power Systems, $26,470-478$.

Mahdavi, M., Monsef, H., \& Romero, R. (2016). Reliability and economic effects of maintenance on TNEP considering line loading and repair. IEEE Transactions on Power Systems, 31, 3381-3393.

Mahdavi, M., Monsef, H., \& Romero, R. (2017). Reliability effects of maintenance on TNEP considering preventive and corrective repairs. IEEE Transactions on Power Systems, 32, 3768-3781.

Romero, R., \& Monticelli, A. (1994). A hierarchical decomposition approach for transmission network expansion planning. IEEE Transactions on Power Systems, 9, 373-380.

Romero, R., Monticelli, A., Garcia, A., \& Haffner, S. (2002). Test systems and mathematical models for transmission network expansion planning. IEE Proceedings-Generation, Transmission and Distribution, 149, 27-36.

Shayeghi, H., \& Mahdavi, M. (2009). Genetic algorithm based studying of bundle lines effect on network losses in transmission network expansion planning. Journal of Electrical Engineering, 60, 237245.

Tor, O. B., Guven, A. N., \& Shahidehpour, M. (2010). Promoting the investment on IPPs for optimal grid planning. IEEE Transactions on Power Systems, 25, 1743-1750. 